\title{
SOME RELATIONS BETWEEN NONEXPANSIVE AND ORDER PRESERVING MAPPINGS ${ }^{1}$
}

\author{
MICHAEL G. CRANDALL AND LUC TARTAR
}

\begin{abstract}
It is shown that nonlinear operators which preserve the integral are order preserving if and only if they are nonexpansive in $L^{1}$ and that those which commute with translation by a constant are order preserving if and only if they are nonexpansive in $L^{\infty}$. Examples are presented involving partial differential equations, difference approximations and rearrangements.
\end{abstract}

Introduction. Let $\Omega$ be a measure space equipped with a nonnegative measure. We write $\int_{\Omega} f$ for the integral over $\Omega$ of $f \in L^{1}(\Omega)$. Some years ago we observed, in a discussion of the Carleman equations (see §3), that if $T$ is a mapping in $L^{1}(\Omega)$ which conserves the integral, i.e.,

$$
\int_{\Omega} T(f)=\int_{\Omega} f
$$

then $T$ is nonexpansive if and only if it is order preserving. To be more precise, let $f \vee g=\max (f, g)$ and $r^{+}=r \vee 0$. We have

Proposition 1. Let $C \subset L^{1}(\Omega)$ have the property that $f, g \in C$ implies $f \vee g \in$ $C$. Let $T: C \rightarrow L^{1}(\Omega)$ satisfy (1) for $f \in C$. Then the following three properties of $T$ are equivalent:

(a) $f, g \in C$ and $f<g$ a.e. implies $T(f)<T(g)$ a.e.,

(b) $\int_{\Omega}(T(f)-T(g))^{+}<\int_{\Omega}(f-g)^{+}$for $f, g \in C$,

(c) $\int_{\Omega}|T(f)-T(g)|<\int_{\Omega}|f-g|$ for $f, g \in C$.

Recently, for all its simplicity, Proposition 1 was useful in the study of difference approximations of scalar conservation laws [2] so we have decided to present it here together with the parallel result for $L^{\infty}(\Omega)$.

Proposition 2. Let $C \subset L^{\infty}(\Omega)$ have the property that $f, g \in C$ implies $f+\|(g-$ $f)^{+} \|_{L^{\infty}(\Omega)} \in C$. Let $T: C \rightarrow L^{\infty}(\Omega)$ satisfy

$$
\text { If } r \in \mathbf{R}^{+}, f \in C \text { and } f+r \in C \text {, then } T(f+r)=T(f)+r \text {. }
$$

Then the following three properties of $T$ are equivalent.

(a) $f, g \in C$ and $f<g$ a.e. implies $T(f)<T(g)$ a.e.,

(b) $(T(f)-T(g))^{+}<\left\|(f-g)^{+}\right\|_{L^{\infty}(\Omega)}$ a.e. for $f, g \in C$,

(c) $|T(f)-T(g)|<\|f-g\|_{L^{\infty}(\Omega)}$ a.e. for $f, g \in C$.

Received by the editors March 6, 1979.

AMS (MOS) subject classifications (1970). Primary 47H99, 35F25, 65M10.

Key words and phrases. Nonlinear operator, order preserving, nonexpansive, rearragement.

'Sponsored by the United States Army under Contract No. DAAG29-75-C-0024.

Supported in part by the National Science Foundation under Grant MCS78-01245.

(C) 1980 American Mathematical Society 0002-9939/80/0000-0120/\$02.50 
These results are proved in $\S 1$ where remarks about variations are also made. Simple but interesting examples are indicated in $\$ 2$.

\section{The proofs.}

Proof of Proposition 1. Assuming that (1) holds we show (a) $\Rightarrow(b) \Rightarrow$ (c) $\Rightarrow$ (a). Let $f, g \in C$. Then $f \vee g=g+(f-g)^{+} \in C$ by assumption and, if (a) holds, $T(f \vee g)-T(g)>0$. Moreover, $T(f)-T(g)<T(f \vee g)-T(g)$. Thus we have $(T(f)-T(g))^{+}<T(f \vee g)-T(g)$. Using this and (1) yields

$$
\int_{\Omega}(T(f)-T(g))^{+}<\int_{\Omega}(T(f \vee g)-T(g))=\int_{\Omega}(f \vee g-g)=\int_{\Omega}(f-g)^{+},
$$

and we have shown (a) $\Rightarrow$ (b). That (b) $\Rightarrow$ (c) is trivial for, assuming (b),

$$
\begin{aligned}
\int_{\Omega}|T(f)-T(g)| & =\int_{\Omega}(T(f)-T(g))^{+}+\int_{\Omega}(T(g)-T(f))^{+} \\
& <\int_{\Omega}(f-g)^{+}+\int_{\Omega}(g-f)^{+}=\int_{\Omega}|f-g| .
\end{aligned}
$$

Finally, if $f, g \in C, f>g$ and (c) holds, the identity $2 s^{+}=|s|+s$ and (1) imply

$$
\begin{aligned}
2 \int_{\Omega}(T(g)-T(f))^{+} & =\int_{\Omega}|T(g)-T(f)|+\int_{\Omega}(T(g)-T(f)) \\
& \leqslant \int_{\Omega}|g-f|+\int_{\Omega}(g-f)=0
\end{aligned}
$$

and so $T(g)<T(f)$ a.e.

Proof of Proposition 2. Assuming that (2) holds we show (a) $\Rightarrow(\mathrm{b}) \Rightarrow$ (c) $\Rightarrow$ (a). Let $f, g \in C$. Then $g+\left\|(f-g)^{+}\right\|_{L^{\infty}(\Omega)} \in C$ by assumption and $g+\|(f-$ $g)^{+} \|_{L^{\infty}(\Omega)}>f \vee g$ a.e. Thus (a) and (2) imply

$$
(T(f)-T(g))^{+}<T\left(g+\left\|(f-g)^{+}\right\|_{L^{\infty}(\Omega)}\right)-T(g)=\left\|(f-g)^{+}\right\|_{L^{\infty}(\Omega)} \quad \text { a.e., }
$$

which is (b). The implication (b) $\Rightarrow$ (c) is immediate as in the previous case. To prove (c) $\Rightarrow$ (a), let $f<g$ a.e. Then using (2) and (c) with $r=\left\|(g-f)^{+}\right\|_{L^{\infty}(\Omega)}=$ $\|g-f\|_{L^{\infty}(\Omega)}$ we have

$$
\begin{aligned}
\|T(f)-T(g)+r\|_{L^{\infty}(\Omega)} & =\|T(f+r)-T(g)\|_{L^{\infty}(\Omega)} \\
& <\|(f-g)+r\|_{L^{\infty}(\Omega)}<r .
\end{aligned}
$$

This implies $T(f)-T(g)<0$ a.e., which is the desired result.

Various generalizations of these results are possible. We next state one of some interest. Let $X, Y$ be vector lattices and $\lambda_{X}, \lambda_{Y}$ be nonnegative linear functionals on $X, Y$ respectively.

Proposition 3. Let $C \subseteq X$ and $f, g \in C$ imply $f \vee g \in C$. Let $T: C \rightarrow Y$ satisfy

$$
\lambda_{Y}(T(f))=\lambda_{X}(f) \text { for } f \in C .
$$

Then (a) $\Rightarrow(\mathrm{b}) \Rightarrow$ (c) where (a), (b), (c) are the properties:

(a) $f, g \in C$ and $f<g$ imply $T(f)<T(g)$,

(b) $\lambda_{Y}\left((T(f)-T(g))^{+}\right)<\lambda_{X}\left((f-g)^{+}\right)$for $f, g \in C$,

(c) $\lambda_{Y}(|T(f)-T(g)|)<\lambda_{X}(|f-g|)$.

Moreover, if $\lambda_{Y}(f)>0$ for $f>0$, then (a), (b), (c) are equivalent. 
The proof is the same as that of Proposition 1. Proposition 2 admits analogous "vector-valued" generalizations. In particular, $T$ may be a mapping $T: C \subset L^{\infty}(\Omega)$ $\rightarrow L^{\infty}\left(\Omega^{\prime}\right)$ with distinct measure spaces $\Omega, \Omega^{\prime}$. In another spirit, if (a) in Proposition 3 is replaced by the requirement that $T+\gamma I$ be order preserving for some $\gamma \in \mathbf{R}^{+}$ and (3) holds, then one deduces that

$$
\lambda_{Y}\left((T(f)-T(g))^{+}\right)<(1+\gamma) \lambda_{X}(f-g)^{+}+\gamma \lambda_{X}(g-f)^{+} .
$$

If (2) in Proposition 2 is replaced by $T(f+r)<T(f)+\gamma r$ for every $r \in \mathbf{R}^{+}$and some $\gamma \in \mathbf{R}^{+}$, then (a) implies that $(T(f)-T(g))^{+}<\gamma\left\|(f-g)^{+}\right\|_{L^{\infty}(\Omega)}$ a.e. Similarly, if (1) is replaced by $\int_{\Omega} T(f+h)<\int_{\Omega} T(f)+\gamma \int_{\Omega} h$ for $h>0$ and some $\gamma \in \mathbf{R}^{+}$, then $\int_{\Omega}(T(f)-T(g))^{+}<\gamma \int_{\Omega}(f-g)^{+}$if $T$ is order preserving. These cases (and probably their vector-valued versions) occur in applications.

All the above could be reformulated in terms of the conditions satisfied by $J(h)=T(h+g)-T(g)$ where $g \in C$ is held fixed to obtain variants. For example, if $J$ preserves the integral, then $J(h)^{+}<J\left(h^{+}\right)$for each $h$ implies that $\int_{\Omega} J(h)^{+}<\int_{\Omega} h^{+}$and $\int_{\Omega} J(h)^{-}<\int_{\Omega} h^{-}$for each $h$ which implies that $\int|J(h)|<$ $\int_{\Omega}|h|$ which in turn implies that $J$ preserves the nonnegative (respectively, nonpositive) functions. We will refrain from more remarks of this sort.

2. Examples. We informally indicate a few examples. The origin of these remarks was the system of equations (called the Carleman equations)

$$
\left\{\begin{array}{l}
u_{t}+u_{x}+\left(u^{2}-v^{2}\right)=0 \\
v_{t}-v_{x}+\left(v^{2}-u^{2}\right)=0
\end{array}\right.
$$

which are to be solved subject to initial conditions

$$
\left\{\begin{array}{l}
u(x, 0)=u_{0}(x), \\
v(x, 0)=v_{0}(x)
\end{array}\right.
$$

where $u_{0}, v_{0} \in L^{1}(\mathbf{R})^{+}$. Assuming (as is the case: [4], [6], [7]) that this problem is solvable in a reasonable sense for $u(t, x), v(t, x)$ we have (formally)

$$
\frac{d}{d t} \int_{\mathbf{R}}(u(t, x)+v(t, x)) d x=\int_{\mathbf{R}}\left(u_{t}+v_{t}\right) d x=\int_{\mathbf{R}}\left(-u_{x}+v_{x}\right) d x=0
$$

and so

$$
\int_{\mathbf{R}}(u(t, x)+v(t, x)) d x=\int_{\mathbf{R}}\left(u_{0}(x)+v_{0}(x)\right) d x .
$$

Letting $\lambda: L^{1}(\mathbf{R}) \times L^{1}(\mathbf{R}) \rightarrow \mathbf{R}$ be given by $\lambda(f, g)=\int_{\mathbf{R}}(f+g) d x$. (6) means that $\lambda\left(S(t)\left(u_{0}, v_{0}\right)\right)=\lambda\left(u_{0}, v_{0}\right)$, where $S(t)$ is the semigroup associated with (4), (5). That is, $S(t)\left(u_{0}, v_{0}\right)=(u(t, \cdot), v(t, \cdot))$. Hence, by Proposition 3, $S(t)$ is order preserving if and only if it is nonexpansive in $L^{1}(\mathbf{R})^{+} \times L^{1}(\mathbf{R})^{+}$(and it is both).

We next briefly illustrate in a simple case the relation to [2]. Consider the problem $u_{t}+f(u)_{x}=0$ where $f: \mathbf{R} \rightarrow \mathbf{R}$ is $C^{1}$. The Lax-Friedrich's difference approximation to this equation is

$$
U_{j}^{n+1}=U_{j}^{n}-\frac{\Delta t}{2 \Delta x}\left(f\left(U_{j+1}^{n}\right)-f\left(U_{j-1}^{n}\right)\right)+\frac{1}{2}\left(U_{j+1}^{n}+U_{j-1}^{n}-2 U_{j}^{n}\right),
$$


which we rewrite as $U^{n+1}=G\left(U^{n}\right)$ where $G$ maps sequences $U=\left\{U_{j}\right\}_{j=-\infty}^{\infty}$ to sequences according to

$$
G(U)_{j}=U_{j}-\frac{\Delta t}{2 \Delta x}\left(f\left(U_{j+1}\right)-f\left(U_{j-1}\right)\right)+\frac{1}{2}\left(U_{j+1}+U_{j-1}-2 U_{j}\right)
$$

Let $l_{1}$ be the space of summable sequences $\left\{U_{j}\right\}_{j=-\infty}^{\infty}$ with the usual ordering, $a<0<b$, and $C=\left\{U \in l_{1}: a<U_{j}<b\right.$ for all $\left.j\right\}$. Clearly

$$
\sum_{j=-\infty}^{\infty} G(U)_{j}=\sum_{j=-\infty}^{\infty} U_{j}
$$

Moreover, $G$ is clearly order preserving on $C$ if $1>(\Delta t / \Delta x)\left|f^{\prime}(r)\right|$ for $a<r<b$. Hence, by Proposition $1, G$ is also nonexpansive in this case.

Next consider the initial value problem

$$
\begin{cases}u_{t}+f(\operatorname{grad} u)=0, & t>0, x \in \mathbf{R}^{N}, \\ u(0, x)=u_{0}(x), & x \in \mathbf{R}^{N},\end{cases}
$$

where $f: \mathbf{R}^{N} \rightarrow \mathbf{R}$. From the form of (7) one expects that if $S(t)$ is the associated semigroup (see, e.g., [3], [5], [10]) then $S(t)\left(u_{0}+r\right)=S(t) u_{0}+r$ for each $r$, and this is indeed the case. Hence we have a quite nontrivial mapping with the property (2). It is both order preserving and nonexpansive in $L^{\infty}\left(\mathbf{R}^{N}\right)$, and one property follows from the other via Proposition 2. The results analogous to [2] for this case are being developed, as are analogous results for equations of the class $u_{t}-\Delta \varphi(u)$ $=0$ where $\varphi: \mathbf{R} \rightarrow \mathbf{R}$ is nondecreasing. (The associated semigroup here often satisfies (1) or its generalizations.)

We offer nonincreasing rearrangements as our last example. Let $\Omega$ be a measure space with $\mu$ the associated measure and $\nu$ be a Borel measure on $(0, \infty)$ such that $r \rightarrow \nu((0, r))$ is a homeomorphism of $(0, \infty)$. If $f>0$ is measurable on $\Omega$ then there is exactly one right-continuous nonincreasing function $f^{*}:(0, \infty) \rightarrow[0, \infty]$ for which

$$
\nu\left\{r \in(0, \infty): f^{*}(r)>\alpha\right\}=\mu\{\omega \in \Omega: f(\omega)>\alpha\}
$$

for $\alpha>0$. This $f^{*}$ is called the nonincreasing rearragement of $f$ (with respect to $\nu$ ). Two particular cases of interest are $\nu((0, r))=r$ (Lebesgue measure) and $\nu((0, r))=$ $c_{N} r^{N}$ where $c_{N}$ is the volume of the unit ball in $\mathbf{R}^{N}$ (see, e.g., [8, p. 184], [9, p. 189]). In the latter case $f^{*}$ can be regarded as a decreasing radial function on $\mathbf{R}^{N}$ with the same distribution function as $f$. Define $T(f)=f^{*}$. It is immediate from the definition that $T$ is order preserving. Moreover, for every continuous function $g$ : $\mathbf{R}^{+} \rightarrow \mathbf{R}^{+}$we have

$$
\int_{0}^{\infty} g(T(f)) d \nu=\int_{\Omega} g(f) d \mu
$$

since $T(f)$ and $f$ have the same distribution function. (Both sides of (8) may be $+\infty$.) Thus $T$ takes $L^{p}(\mu)^{+}$to $L^{p}(\nu)^{+}$for $1<p<\infty$ and preserves the integral. Moreover it is immediate from the definition that $T(f+c)=T(f)+c$ for $c \in$ $\mathbf{R}^{+}$. Hence $T$ is nonexpansive from $L^{p}(\mu)^{+}$to $L^{p}(\nu)^{+}$for $p=1, \infty$. In fact, if

$$
j:[0, \infty] \rightarrow[0, \infty] \text { is convex, lower semicontinuous and } j(0)=0 \text {, }
$$


then

$$
\int_{0}^{\infty} j\left((T(f)-T(g))^{+}\right) d \nu<\int_{\Omega} j\left((f-g)^{+}\right) d \mu
$$

whenever $f, g>0$ and $\int_{\Omega} j(f) d \mu, \int_{\Omega} j(g) d \mu<\infty$. This follows from a variant of a result of Brezis and Strauss [1].

Proposition 4. Let $\Omega, \Omega^{\prime}$ be measure spaces with measures $\mu, \nu$ respectively. Let $K$ : $L^{1}(\mu)^{+} \rightarrow L^{1}(\nu)^{+}$satisfy

$$
\int_{\Omega^{\prime}}|K(f)-K(g)| d \nu<\int_{\Omega}|f-g| d \mu \quad \text { for } f, g \in L^{1}(\mu)^{+}
$$

and

$$
K(f)<\|f\|_{L^{\infty}(\mu)} \text { a.e. } \nu \text { for } f \in L^{1}(\mu)^{+} \text {. }
$$

Then for each $j$ as in (9) and $f \in L^{1}(\mu)^{+}$,

$$
\int_{\Omega^{\prime}} j(K(f)) d \nu<\int_{\Omega} j(f) d \mu
$$

The idea of the proof is as follows: Let $t>0, f \in L^{1}(\mu)^{+}$and set $h=f \wedge t$. Then $h<t$ and so $K(h) \leqslant t$ a.e. $\nu$ by (12). Hence $(K(f)-t)^{+}<(K(f)-K(h))^{+}$ and so, by (11),

$$
\int_{\Omega^{\prime}}(K(f)-t)^{+} d \nu<\int_{\Omega^{\prime}}(K(f)-K(h))^{+} d \nu<\int_{\Omega}|f-h| d \mu=\int_{\Omega}(f-t)^{+} d \mu .
$$

Next one integrates this inequality with respect to the measure $d j^{\prime}(t)$ and uses (properly interpreted) the identity

$$
j(r)=\int_{0}^{\infty}(r-t)^{+} d j^{\prime}(t)+r j^{\prime}(0+)
$$

to find (13).

To apply Proposition 4 to prove (10) we fix $g \in L^{1}(\mu)^{+} \cap L^{\infty}(\mu)^{+}$and set $K(f)=(T(f)-T(g))^{+}$. Then (11) and (12) follow from what we have shown above. Thus (10) holds for $f \in L^{1}(\mu)^{+}$and $g$ as above. For the general case, choose $f_{n}, g_{n} \in L^{\infty}(\mu)^{+} \cap L^{1}(\mu)^{+}$increasing to $f, g$ as $n \rightarrow \infty$, note that $j\left(\mid f_{n}-\right.$ $\left.g_{n} \mid\right)<j(f) \vee j(g)$ and use the dominated convergence theorem and Fatou's lemma.

It seems unlikely that $(10)$ is not in print, but we do not know a reference. $R$. Turner points out that it may be deduced from approximation by simple functions in a straightforward (but not simpler) way. Here it is exhibited as a special case of general facts. We also felt it worthwhile to recall the useful and simple ideas represented by Proposition 4 and its proof, as they are perhaps not as well known as they deserve to be. 


\section{REFERENCES}

1. H. Brezis and W. Strauss, Semilinear elliptic equations in $L^{1}$, J. Math. Soc. Japan 25 (1973), 15-26.

2. M. G. Crandall and A. Majda, Monotone difference approximations for scalar conservation laws, Math. Comp. (to appear).

3. A. Douglis, Lectures on discontinuous solutions of first order nonlinear partial differential equations in several space variables, North British Symposium on Partial Differential Equations, 1972.

4. Ignace I. Kolodner, On the Carleman's model for the Boltzmann equation and its generalizations, Ann. Mat. Pura Appl. 63 (1963), 11-32.

5. S. N. Kružkov, Generalized solutions of first order nonlinear equations in several independent variables, Mat. Sb. 70 (1966), 394-415.

6. T. Kurtz, Convergence of sequences of semigroups of nonlinear operators with an application to gas kinetics, Trans. Amer. Math. Soc. 186 (1973), 259-272.

7. M. Pierre, Un théorème de generation de semi-groupes nonlinéaires, Israel J. Math. 23 (1976), 189-199.

8. G. Pólya and G. Szegö, Isoperimetric inequalities in mathmematical physics, Ann. of Math. Studies, no. 27, Princeton Univ. Press, Princeton, N.J., 1951.

9. E. Stein and G. Weiss, Introduction to Fourier analysis on Euclidean spaces, Princeton Univ. Press, Princeton, N.J., 1971.

10. M. B. Tamburro, The evolution operator approach to the Hamiltion-Jacobi equation, Israel J. Math. 26 (1977), 232-264.

Department of Mathematics and Mathimatics Research Center, University of WisconSiN-MAdison, MAdison, Wisconsin 53706

Department of Mathematics, Universtié de Paris-Sud, Centre d'Orsay, Orsay, France 